\title{
Adaptive Thermal Comfort for Occupants of Low-Cost Dwellings in a Hot Dry Climate
}

\author{
Irene Marincic ${ }^{1}$, J. Manuel Ochoa ${ }^{1}$, M. Guadalupe Alpuche ${ }^{1}$ and Gabriel Gómez-Azpeitia ${ }^{2}$ \\ ${ }^{1}$ Department of Architecture and Design, University of Sonora, Hermosillo 83000, Mexico \\ ${ }^{2}$ Faculty of Architecture and Design, University of Colima, Colima 28400, Mexico
}

\begin{abstract}
Adaptive models are based on the observation that there are some actions that people can and actually do take to achieve thermal comfort. Studies regarding thermal comfort conditions in economical dwellings were carried out simultaneously in seven Mexican cities, corresponding to warm dry and warm humid climates. In this article, case studies of low-cost dwellings in the city of Hermosillo (in northwest Mexico), are presented and analyzed. Field surveys were carried out to obtain information about the physical characteristics of the dwellings and their occupants, as well as the indoor thermal environment. Neutral temperature was obtained from the applied survey. The high neutral temperature reveals the effect of inhabitants' adaptation mechanism to extreme climates. Occupant comfort votes as a function of indoor air temperatures were analyzed, and different characteristics such as age, size and gender were evaluated separately. The results show the variability of the neutral temperature and the tolerance to temperature changes, depending on the population's specific characteristics. In many cases where the population does not have access to artificial acclimatization devices, the neutral temperature values for specific climates and people can inform architects when choosing the most suitable thermal strategies for building design.
\end{abstract}

Key words: Adaptive comfort, low-cost dwelling, hot dry climate.

\section{Introduction}

Thermal comfort studies have been carried out all around the world by many authors, and some adaptive comfort models have been developed and proven specifically for warm climates, such as Auliciems and de Dear [1], Heidari and Sharples [2], among others [3]. Adaptive models are based on the observation of actions that people can and actually do take to achieve thermal comfort. Acclimation and other psychological issues are involved, and the influence of these variables on thermal comfort is more evident in a naturally ventilated building. This approach recognizes that people can try to achieve their thermal comfort inside buildings by modifying their schedules, clothing, and manipulating their environment between certain limits. There are different physiological and psychological adaptation levels [4] that contribute to achieving

Corresponding author: Irene Marincic, $\mathrm{PhD}$, research fields: thermal behaviour of buildings, thermal comfort, daylighting. E-mail: imarincic@arq.uson.mx. comfort conditions. Expectation, previous experience, spatial conditions and ability to control environment stimuli [5] are important variables to be considered.

In Mexico, the need for quick and inexpensive construction of dwellings for underprivileged people means that in any climate, housing developments are designed and constructed without regional adaptation, using almost the same model for the whole country. In extreme climates like that of northwest Mexico, indoor thermal conditions are not acceptable during most of the year, and excessive energy for air conditioning is used, leading to high acclimatization costs. Also, a high number of people cannot afford the cost of air-conditioning systems and must try to adapt to high temperature environments.

In the context of an inter-institutional project supported by Mexican federal funds, several studies on thermal comfort conditions in low-cost dwellings were carried out simultaneously in seven cities corresponding to warm dry and warm humid climates 
throughout the Mexican territory [6]. In this article, case studies of low-cost dwellings in the city of Hermosillo (in northwest Mexico) are presented and analyzed. In this city, the climate is hot dry; despite this condition, a very high number of houses are not designed or constructed to suit the extreme local climate. Through field studies, physical characteristics of houses and inhabitants, as well as indoor climatic variables and occupants' reported thermal sensation were sampled. Indoor thermal variables were measured and a questionnaire was applied to occupants simultaneously. Most of the inhabitants are habituated to the local climate. Inhabitants' thermal sensation was evaluated in their own habitat, where they are able to take steps to seek their thermal comfort.

The neutral temperature ( $\mathrm{Tn}$ ) was obtained from the applied survey, taking into consideration environmental conditions and particular occupants' characteristics. The high neutral temperature values reveal the effect of people's adaptation mechanism to extreme climates.

\section{Local Climate and Surveys}

\subsection{Climate}

The local climate is characterized by high solar radiation levels, clear skies throughout the year and large daily and seasonal temperature oscillations. Summers are very hot, with daily temperatures between 15 and $21^{\circ} \mathrm{C}$ and 40 to $45^{\circ} \mathrm{C}$, and relative humidity between 50 and $15 \%$. Summer wind is usually warm, so it is inadequate for passive cooling or for better outdoor thermal comfort. Winters are mild, with minimum temperatures from 0 to $7^{\circ} \mathrm{C}$ and maximum temperatures between 25 and $30^{\circ} \mathrm{C}$. Because of the extreme conditions during the summer, part of the population is habituated to air conditioning, but people with lower incomes generally have no access to these devices and cannot afford their operation costs. This segment of society often lives in small houses within large developments, constructed in many cases without insulation, so indoor thermal conditions are not comfortable at all, particularly in the summer.

In Figs. 1 and 2, indoor temperature and relative humidity during the winter and summer survey periods are plotted. The measures correspond to the surveyed houses analyzed in the following paragraphs.

In the next graph (Fig. 3), comfort votes for both periods are summarized as a percentage of the total votes for each period. The percentage has been obtained considering the number of thermal sensation votes from each level (ASHRAE scale from -3 to +3 ) divided by the total number of responses from each survey period. The periods have been calculated separately but the results have been plotted together in the graph.

As can be seen in Figs. 1 and 2, winter indoor conditions are mild and it is not so difficult to adapt to

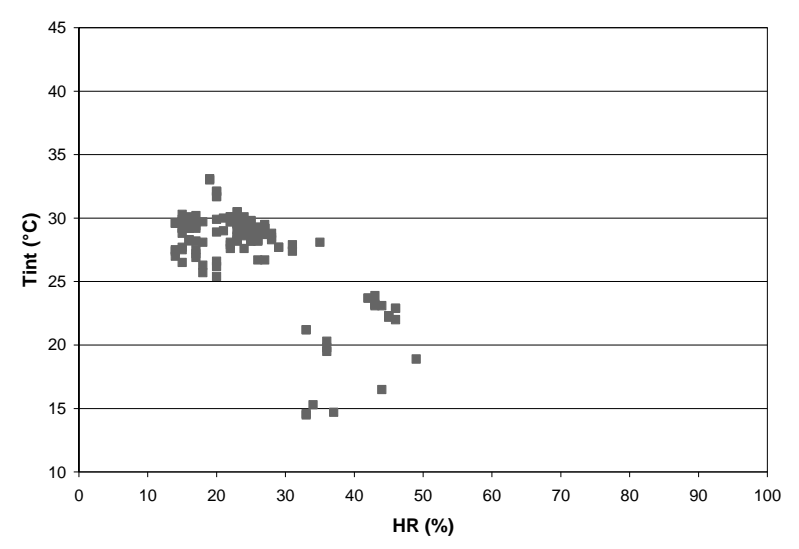

Fig. 1 Relative humidity and air temperature indoors during the winter survey period.

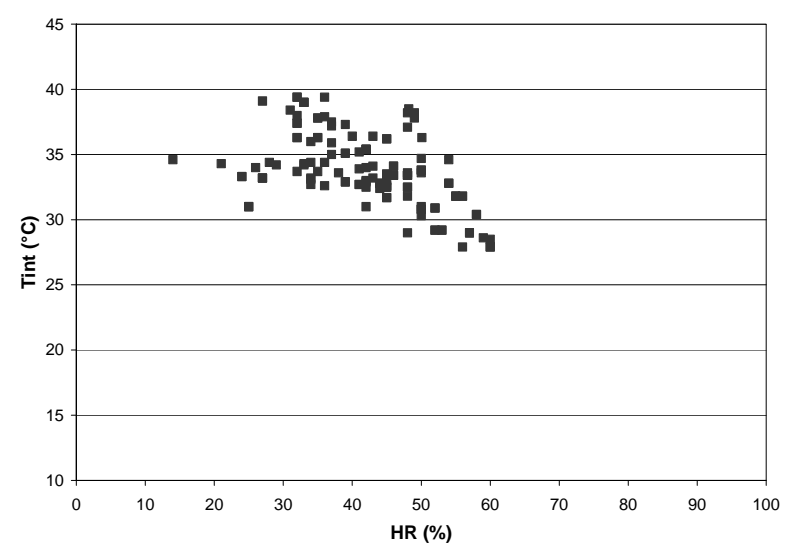

Fig. 2 Relative humidity and air temperature indoors during the summer survey period 


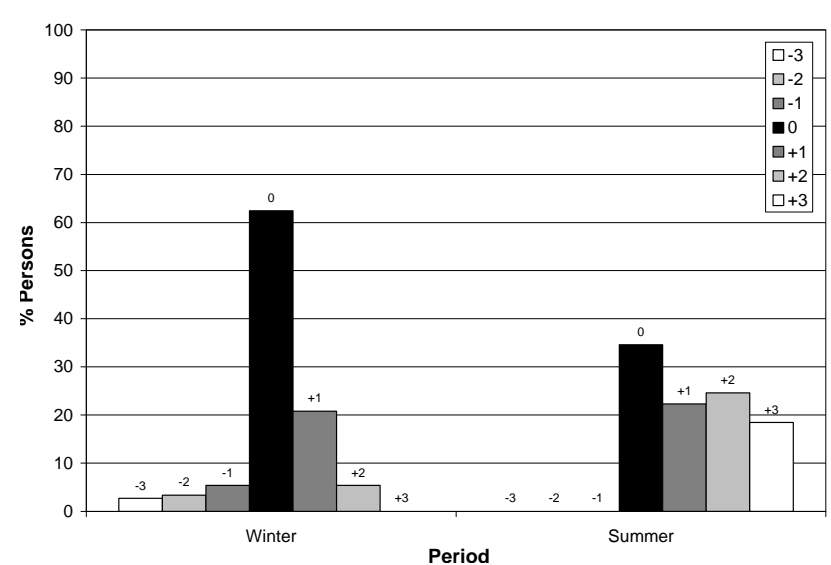

Fig. 3 Thermal sensation votes during winter and summer survey periods (\% of each vote level per period).

indoor climates. In Fig. 3, more than $60 \%$ of sensation votes express comfort or zero value in the scale. However, summer indoor conditions are not very hospitable, as expressed in the percentage of high sensation votes (from +1 to +3 ) in Fig. 3 .

\subsection{Field Surveys}

By applying field surveys, it is possible to analyze occupants' thermal sensations in their own environment, obtaining data at the site where they live or work. This type of comfort study enables the evaluation of the physiological and psychological responses of inhabitants as a whole, including the actions they take to achieve thermal comfort. They also allow the prediction of occupants' thermal sensation and comfort temperature, which vary at least with the climate and the season.

The comfort studies presented here consisted of field surveys in several low-cost housing developments in the city of Hermosillo, Mexico (Fig. 4). Occupants of the surveyed houses were workers with very low income, the majority habituated to the local climate. They were generally families made up of young couples with one or two children. Houses were constructed in 33.5 to $39 \mathrm{~m}^{2}$, distributed in two main spaces — one bedroom and one multi-purpose room — and one bathroom. The houses were made of concrete block walls, joist slabs and polystyrene vaults, without insulation.
Field studies consisted of sampling physical characteristics of the houses, social and physical characteristic of the occupants, and reported thermal sensation from the occupants. This was achieved by applying a questionnaire specifically designed for this purpose. During survey application, indoor thermal variables were measured (Fig. 5).

The information obtained about the dwellings was, for example: house orientation, physical characteristics and geometry, materials, air conditioning and ventilation devices, and shadow devices, among others. Information collected about the occupants included age, gender, weight, height and socio-economic data (such

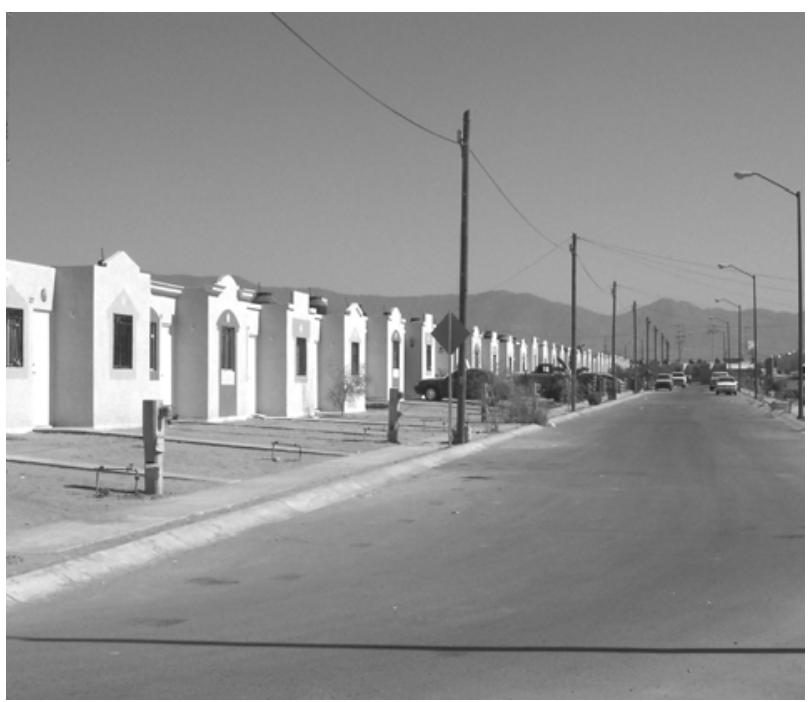

Fig. 4 One of the tract housing developments surveyed.

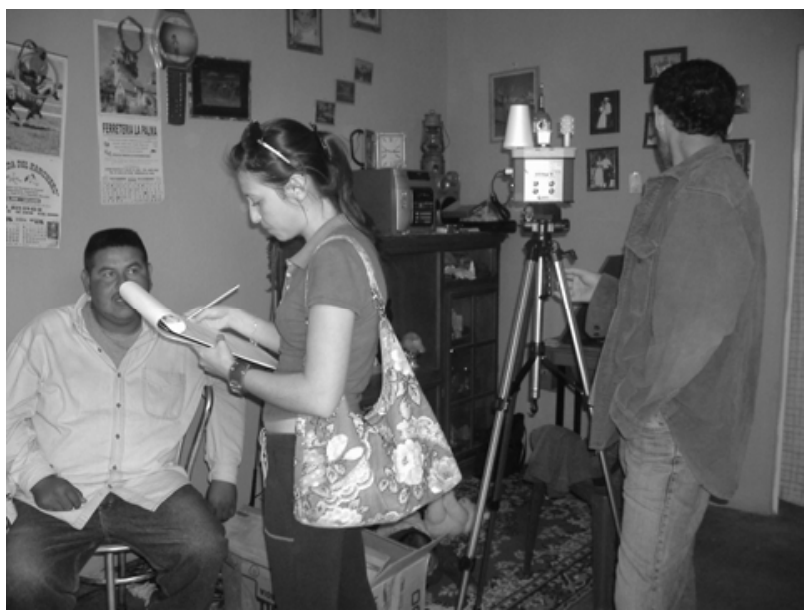

Fig. 5 Field surveys in houses. A student is applying a questionnaire while indoor thermal conditions are measured. 
as education level, salary, etc.). Clothing and activity level were reported in each case. In the field survey, the reported thermal sensations were based on a seven-point scale (ASHRAE scale from -3 to +3 ), representing occupant comfort votes. At the same time, the following thermal variables were measured indoors: air temperature, globe temperature, relative humidity and wind speed. Considering local climate characteristics, the surveys were carried out during the two most relevant periods: winter and summer. The survey questionnaire and its application methodology were designed according to ISO 7730 and ISO 10551 standards $[7,8]$. The measurement devices complied with ISO 7726 standards [9]. The study sample was determined based on the amount of this type of housing built in the city and included at least 150 people per season. Indoor climatic variables were obtained with the QUESTemp ${ }^{\circ} 36$ thermal environmental monitor (Fig. 6), which is a data logger with several sensors able to measure the following variables: dry bulb temperature, wet bulb temperature, black globe temperature, relative humidity, and wind speed. Meteorological data were obtained from the meteorological station of the Energy, Environment and Architecture Laboratory at the University of Sonora, located in Hermosillo.

Indoor thermal measurements were collected after being in the same environment for a few minutes, so that devices could be ready to take appropriate data.

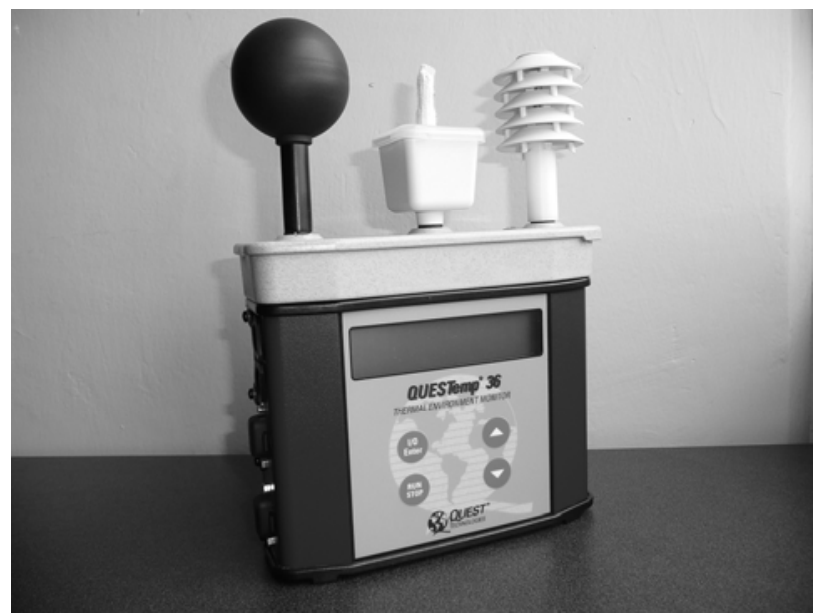

Fig. 6 Thermal environmental monitor.

\section{Results and Interpretation}

\subsection{Sensation Votes and Neutral Temperature}

By analyzing the five measured indoor variables, we observed that in this case air temperature and globe temperature were the most relevant variables affecting sensation votes. We compared both (Fig. 7) in order to select the most appropriate data to work with. Although field surveys were carried out in two periods (summer and winter), the subsequent graphs present results only for the most critical adaptation period: summer.

In Fig. 7, indoor air temperature (Tint) vs. globe temperature (Tg) are plotted. Each pair of simultaneous data is represented by black triangles, and the black line is the linear regression of these data. The gray line has been plotted to compare both variables, and is a hypothetical line where both temperatures are equal.

Fig. 7 is plotted in order to compare how much air temperature differs from globe temperature. As can be observed, during this period both are almost proportional, which indicates that both are able to be used as the most relevant indoor variables which impact on thermal sensations. We have chosen air temperature because this makes it easier to compare our results with other authors', who mostly use air temperature.

Hermosillo's climate can be considered "asymmetric", according to Nicol [10]. In such

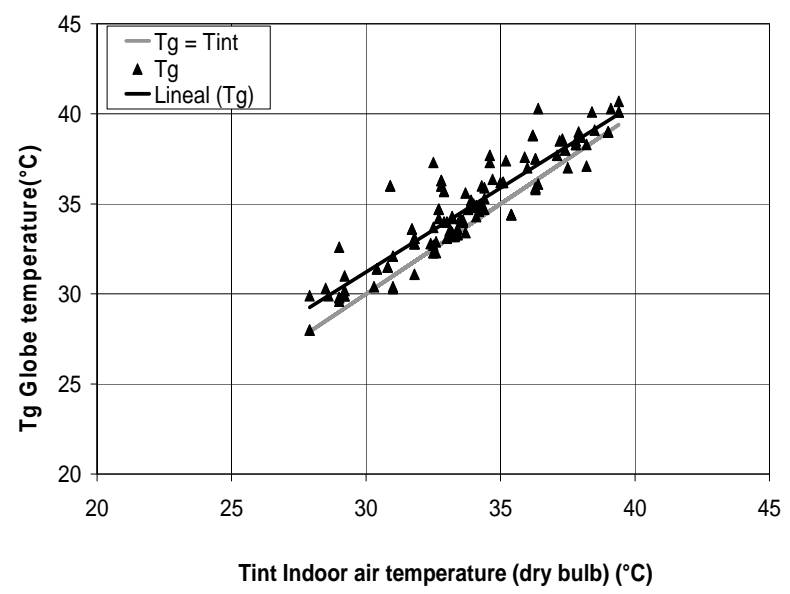

Fig. 7 Air temperature vs. globe temperature indoors during the summer survey period (black triangles and black line). Gray line is $\mathbf{T g}=$ Tint. 
climates, thermal sensation responses during field studies show a tendency toward only one extreme of the sensation scale. In this case, the "warm" votes have no symmetric response at the opposite side of the scale (could). Therefore, the conventional regression method distorts the results. In order to estimate the neutral temperature (Tn) and its limits (comfort range), a method of statistical regression by layers was utilized, to avoid the characteristic bias of asymmetric climates [11]. The method is based on Nicol's proposal for "asymmetric" climates [10]. The fundamental difference with the conventional method is that instead of obtaining the regression line that characterizes the complete sample studied, several layers are grouped by level of thermal sensations expressed $(+1,+2, \ldots)$. If the number of responses for each level is not enough to obtain reliable results, data for that level are not considered. Subsequently, average temperature and standard deviation per layer was obtained. The intersection between the regression line obtained with the average values and the 0 -line sensation vote (sensation of thermal neutrality) determines the value of the neutral temperature (Tn). The addition and the subtraction of the standard deviation $(\sigma)$ for each layer Tn $\pm \sigma$ determines the limits of the comfort range.

In Fig. 8, averaged sensation votes from the whole survey are plotted with the corresponding indoor air temperatures. The figure shows the comfort or neutral temperature (Tn), which in this case is $32.2^{\circ} \mathrm{C}$ for summer. The calculated comfort range (close range) is $\operatorname{Tn} \pm 2.45^{\circ} \mathrm{C}$, which encompasses $76 \%$ of the votes. For the winter period (not presented here) we obtained $\mathrm{Tn}=$ $26.9^{\circ} \mathrm{C}$. The Tn value seems to be very high, compared to the results reported in the literature, but this evidences the adaptation mechanism of people to extreme climates. Also, the wide comfort range is a function of the wide external temperature oscillations in hot dry climates.

Although many variables are not easy to isolate, the following paragraphs describe comfort votes as a function of indoor temperature, depending on different people's characteristics, such as age, body size and gender.

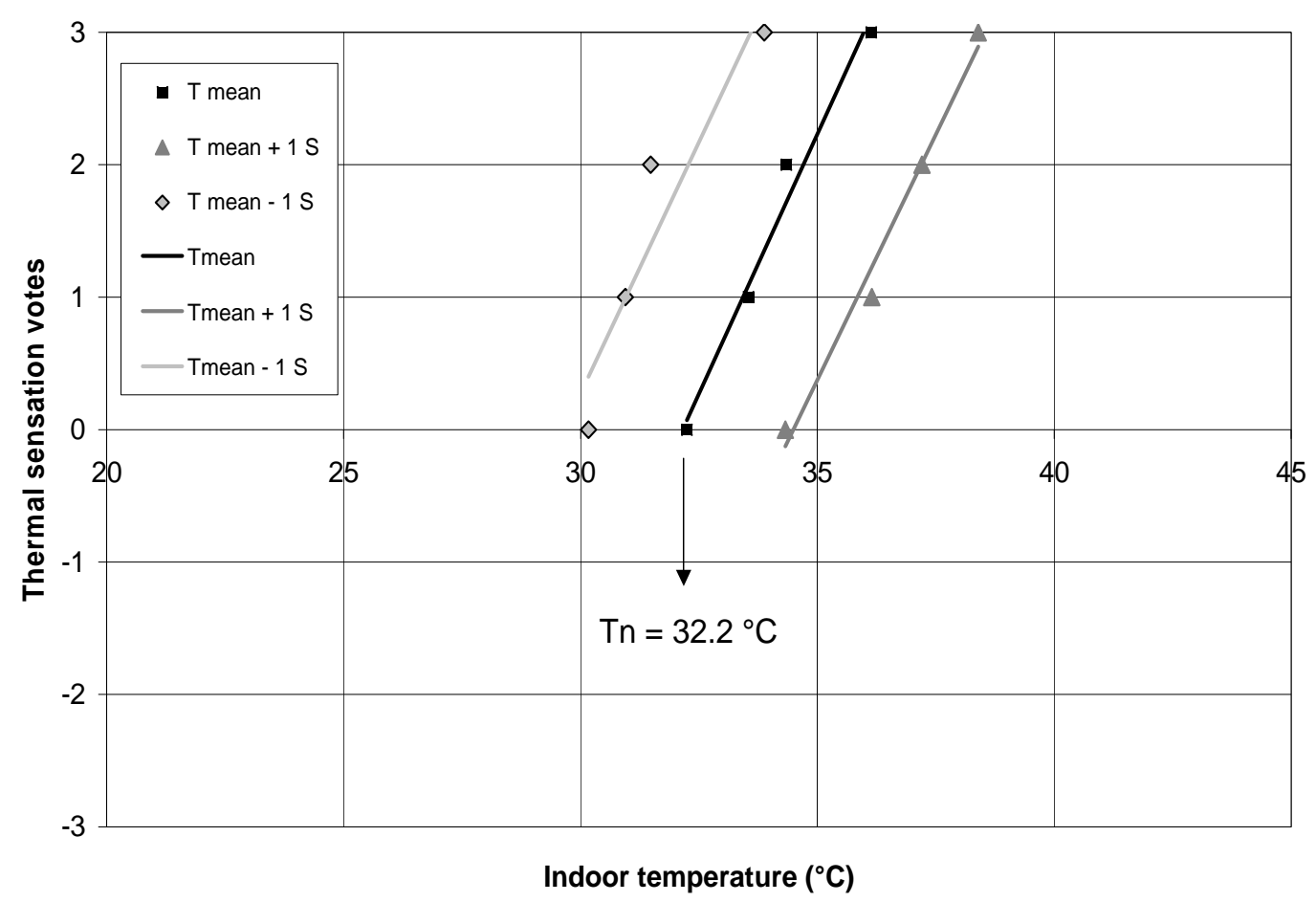

Fig. 8 Thermal sensation votes as a function of indoor air temperature during summer survey period (in the graph the symbol S corresponds to $\sigma)$. 


\subsection{Age}

Fig. 9 illustrates the influence of people's age on sensation votes. The typical inhabitants of these houses are young couples with one or two children (usually one). No children participated in the applied comfort survey questionnaires. People under 40 years of age represented $85 \%$ of the sampled population and $15 \%$ were people over 40 . Considering the age distribution in this population, we split them into two groups, one below 40 years of age and the other above 40 . The tendency in Mexico is towards an inversion of the population pyramid, which means a higher number of older people in the future. In order to analyze two different age groups, we set the limit at 40 years in order to have enough data in both groups. Sensation votes from both groups are plotted separately in Fig. 9.

The two age groups seemed to have similar responses to thermal effects (similar slope in the regression line), but the older group seemed to feel more comfort with higher temperatures. In older people, thermoregulatory mechanisms are less efficient, and activity habits and capacity are decreased. In our survey, this last factor was probably the most relevant and explains our results, since less activity is associated with lower thermal sensations.

\subsection{Body Size}

The surveyed occupants, and local inhabitants in general, tend to be stout people. We used body mass index (BMI) as an indicator, instead of using only weight. In our case, $58 \%$ of the occupants had a body mass index higher than 25 (a 25 BMI is usually considered as the upper "normal" size limit). Official population data report that $67.6 \%$ of men and $77.5 \%$ of women older than 20 years in the region (the State of Sonora, Mexico) have a BMI greater than 25 [12].

In Fig. 10, both groups are plotted separately. At relatively moderate temperatures, both groups seemed to have similar sensations as a result of thermal conditions (in this case thinner people preferred a slightly lower Tn), although the different slopes in the regression lines indicate that bigger people had less tolerance to temperature changes. At temperatures higher than about $34^{\circ} \mathrm{C}$, as expected, bigger people preferred lower temperatures, and they arrived at the sensation of "hot" $(+3)$ at lower temperatures than thinner persons. The reason is that, in bigger people, increased metabolic rate (due to the increase in volume) is proportionally higher than the increase in skin surface, which does not allow enough heat dissipation to balance the heat exchange.

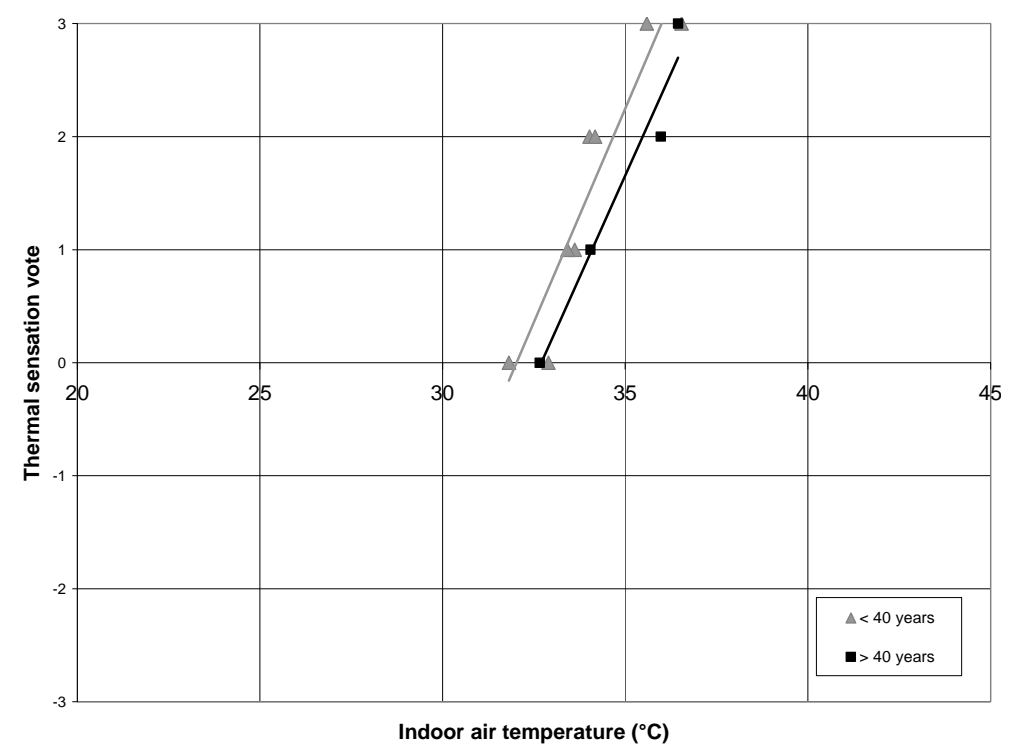

Fig. 9 Thermal sensation votes as a function of indoor air temperature during summer survey period (depending on people's age). 


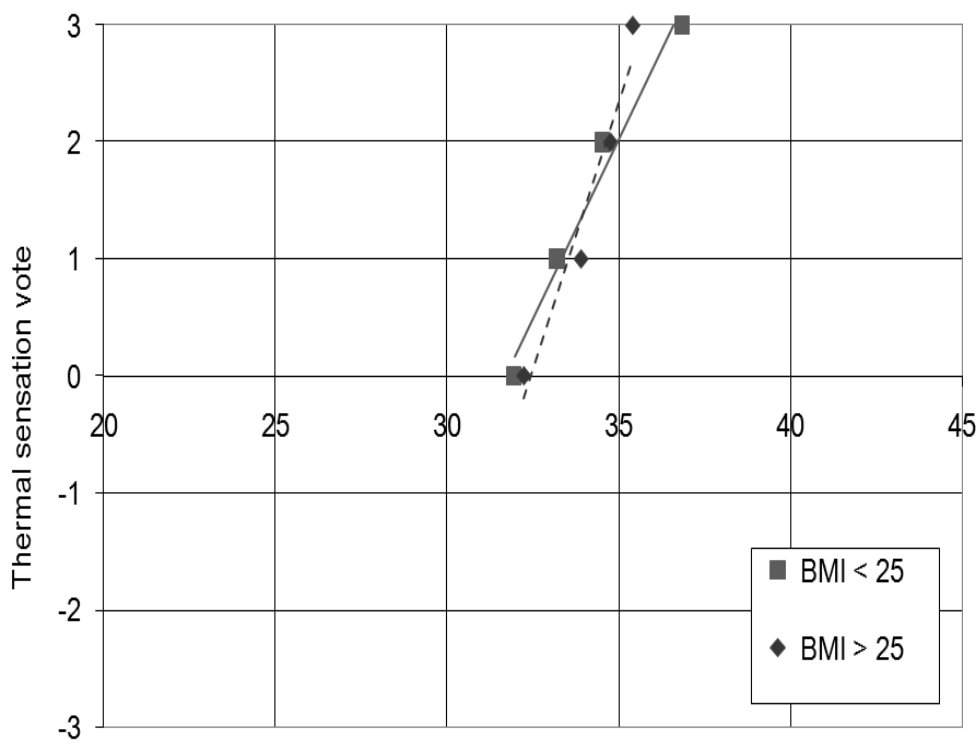

Indoor air temperature $\left({ }^{\circ} \mathrm{C}\right)$

Fig. 10 Thermal sensation votes as a function of indoor air temperature during summer survey period (depending on people's body mass index BMI).

\subsection{Gender}

The gender distribution in the field survey was almost evenly divided between females and males. In Fig. 11, females and males are plotted separately, showing slightly different responses to temperature.

The graph shows that at relatively moderate temperatures, females and males had a similar response

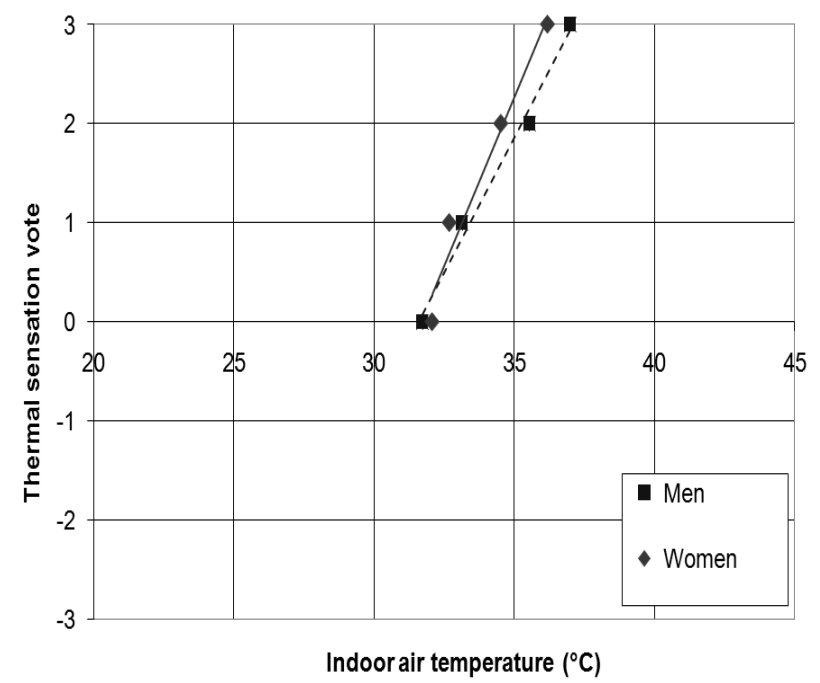

Fig. 11 Thermal sensation votes as a function of indoor air temperature during summer survey period (depending on people's gender). to temperature. As temperature increased, females preferred lower temperatures (in warm climate) than males and showed less tolerance to temperature changes. Women are physiologically more sensitive to temperature changes and have a slightly lower thermal adaptation capacity.

\section{Conclusion}

The results of the field survey presented in this article give a good idea of the complexity of people's sensation of thermal environments, and can help to determine neutral temperatures under different circumstances. Field surveys are also helpful for developing models to understand the mechanisms that influence thermal comfort in extreme climates.

In this article, results show a variability of neutral temperature and tolerance to temperature changes, depending on the specific characteristics of the people surveyed. Field surveys are a useful methodology for detecting and studying thermal adaptation phenomena. The understanding of neutral temperatures, based on many variables involved in human sensation, is useful for an optimal control of air conditioning systems, which leads to better thermal conditions for the 
occupants and a conscious utilization of energetic resources for cooling. In many cases within Mexico, where the population does not have access to artificial acclimatization devices (despite extreme climate conditions), neutral temperature values can guide architects when choosing the most suitable thermal strategies for building design, allowing them to obtain indoor thermal conditions closest to the comfort conditions of the specific population and climate. Furthermore, lowering indoor temperature values with the application of passive cooling strategies can help occupants achieve a better quality of life.

\section{Acknowledgments}

The project "Comfort and thermal energy efficiency in low-cost dwellings in Mexico: regions of warm dry and warm humid climates" was supported by Mexican federal funds from the National Housing Council (CONAVI) and the National Council of Science and Technology (CONACyT).

\section{References}

[1] A. Auliciems and R. de Dear, Air-conditioning in Australia I - Human thermal factors, Architectural Science Review 29 (3) (1978) 67-75.

[2] S. Heidari and S. Sharples, A comparative analysis of short-term and long-term thermal comfort surveys in Iran, Energy and Buildings 34 (2002) 607-614.

[3] G. S. Brager and R. J. de Dear, Thermal adaptation in the built environment: A literature review, Energy and Buildings 27 (1998) 83-96.
[4] R. De Dear, G. Brager and D. Cooper, Developing and adaptive model of thermal comfort and preferences (Final Report on RP-884), ASHRAE and Macquaire Research Ltd., 1998.

[5] M. Nikolopoulou and K. Steemers, Thermal comfort and psychological adaptation as a guide for designing urban spaces, Energy and Buildings 35 (2003) 95-101.

[6] I. Marincic, J. M. Ochoa, M. G. Alpuche and G. Gómez-Azpeitia, Adaptive thermal comfort in warm dry climate: Economical dwellings in Mexico, in: 26th Conference on Passive and Low Energy Architecture PLEA 2009, Quebec City, Canada, 2009, pp. 510-515.

[7] International Organization of Standardization ISO 7730:2005 (E) Ergonomics of the thermal environment analytical determination and interpretation of thermal comfort using calculation of PMV and PPD indices and local thermal comfort criteria, 2005.

[8] International Organization of Standardization ISO 10551:1995 (E) Ergonomics of the thermal environment assessment of the influence of thermal environment using subjective judgment scales, 1995.

[9] International Organization of Standardization ISO 7726:1998 (E) Ergonomics of the thermal environment instruments for measuring physical quantities, 1998.

[10] F. Nicol, Thermal Comfort: A Handbook for Field Studies toward an Adaptive Model, London, University of East London, 1993.

[11] G. Gómez-Azpeitia et al., Comfort Temperatures inside Low-Cost Housing, in: 26th Conference on Passive and Low Energy Architecture PLEA 2009, Quebec City, Canada, 2009, pp. 498-503.

[12] Instituto Nacional de Salud Pública, Encuesta Nacional de Salud y Nutrición 2006, Cuernavaca, Mexico, 2007. (in Spanish) 